\title{
Food-induced pulmonary hemosiderosis
}

Volume 5 Issue 7 - 2017

\section{Keywords}

Immune deficiencies, Pulmonary infiltrates, Allergy, Pulmonary disease, Pneumonia, Thrombocytosis and hemophagocytic lymphohistiocytosis, Immunology, Infectious disease, Hematology, Diarrhea

\section{Introduction}

Heiner syndrome (food-induced pulmonary hemosiderosis) is an atypical pulmonary disease that is caused by a non-IgE mediated hypersensitivity, primarily to cow's milk. ${ }^{1}$ Cow's milk protein allergy prevalence is $2-7.5 \%$ in infants, but estimated to be $0.5 \%$ in breastfed infants. ${ }^{2}$ It is estimated that $5 \%$ of infants with cow's milk protein allergy have evidence of pulmonary infiltrates. ${ }^{3}$ This case is presented to increase physician awareness of this condition, decrease misdiagnosis with other etiologies of pulmonary infiltrates such as infections and immune deficiencies, and to prevent detrimental consequences that can be easily remedied. ${ }^{1}$

\section{Case presentation}

A two-month-old male with past medical history of recurrent diarrhea and failure to thrive presented with persistent cough, tachypnea and high inflammatory markers despite antibiotic treatment for pneumonia. One week earlier our patient was diagnosed with bilateral pneumonia based on same symptoms as well as chest x-ray findings, and was started on Amoxicillin $80 \mathrm{mg} / \mathrm{kg} /$ day. There was no fever. Patient was born full-term via scheduled cesarean section, with no complications noted during pregnancy or delivery. Since birth, patient experienced a decrease in weight for age percentiles and watery diarrhea. He went through multiple types of formula and following positive stool lactoferrin, he was switched to a partially hydrolyzed formula. His diarrhea improved suggesting the patient had a food protein allergy. Otherwise, patient's family and social history were non-contributory. On exam, patient was a febrile, showed no significant distress, mild nasal congestion with occasional cough and mild intercostals retractions. The chest auscultation was normal. There were no rashes. Remainder of the physical exam was unremarkable. Laboratory results included the following (reference ranges provided parenthetically): Hemoglobin of $9 \mathrm{gm} / \mathrm{dl}$ (9.0-15.0), WBC of $23.4(5.0-19.5 \times 10(9) / \mathrm{L})$, platelets of $919(140-440 \times 10(9) / \mathrm{L})$, C-reactive protein of $33.4 \mathrm{mg} / \mathrm{L}(<6.0)$, erythrocyte sedimentation rate of $>130 \mathrm{~mm} / \mathrm{hr}(0-2)$. Procalcitonin, lactic acid, electrolyte panel, liver enzymes and the rest of the complete blood count (CBC) were unremarkable. A respiratory viral panel was negative. Sweat chloride test was also performed and found to be negative. Aspiration and tracheoesophageal fistula were deemed unlikely after evaluation with swallow study and upper gastrointestinal series with barium that showed lack of aspiration and normal anatomy with contrast staying within the gastrointestinal tract. Patient was started on azithromycin to cover atypical bacteria. Pulmonary infiltrates, tachypnea, high inflammatory markers and thrombocytosis persisted despite antibiotic treatment for pneumonia.

\author{
Jasmine Alsukhon, Ahmed Elisa, Garrett \\ Koon, Andrey Leonov \\ Department of Pediatrics and Adolescent Medicine,Western \\ Michigan University, USA
}

Correspondence: Jasmine Alsukhon, Department of Pediatrics and Adolescent Medicine,Western Michigan University, Homer Stryker MD School of Medicine, 1000 Oakland Dr, Kalamazoo, MI 49008, USA, Email jasmine.alsukhon@med.wmich.edu

Received: July 10, 2017| Published: September 18, 2017
Allergy/immunology, infectious disease, hematology and gastrointestinal consults were done. Primary thrombocytosis and hemophagocytic lymphohistiocytosis (HLH) were excluded by hematology team. From infectious disease, extreme thrombocytosis and leukocytosis was not related to the atypical pneumonia and the underlying cause was unclear. Given the history of possible food protein allergy, ongoing GI inflammation was suspected despite clinical improvement in diarrhea, and this was confirmed by repeating fecal lactoferrin and calprotectin that remained elevated on the partially hydrolyzed formula. With indication of inadequate control of food protein allergy, Heiner's syndrome was suspected and patient was switched to an amino acid-based formula during this hospital admission. Cow's milk IgG4 was found to be elevated to $10.20 \mathrm{mcg} /$ $\mathrm{mL}$ (normal range; $<0.15 \mathrm{mcg} / \mathrm{mL}$ ). Cow's milk IgE was normal (normal range; $<0.35 \mathrm{kU} / \mathrm{L}$ ). Improvement in inflammation and respiratory condition was noted only after switching to the amino acid based formula, suggesting pulmonary hemosiderosis and enteropathy due to milk allergy. Twenty days after discharge WBC, PLT, ESR and $\mathrm{CRP}$ values returned to normal limits. Repeat chest x-ray done at an outside hospital showed progressive improvement.

\section{Discussion}

In our case, differential diagnosis was wide, including pneumonia, aspiration pneumonitis and cystic fibrosis. They were ruled out by the treatment teams given the lack of response to antibiotics, negative sweat test, and normal swallowing study. Clinical improvement with complete elimination of cow's milk protein supported the diagnosis of Heiner's syndrome. Heiner syndrome was first described by Heiner and Sears in 1960. He described seven cases with precipitins and positive intradermal tests to cow's milk, four of whom had features of pulmonary hemosiderosis. Symptoms disappeared with a milk-free diet. ${ }^{4}$ In a case study done in 2005 by Ioannis Moissidis which reviewed eight cases, all presented with upper respiratory 
tract symptoms in the form of cough, wheezing, hemoptysis, nasal congestion, dyspnea, recurrent otitis media, recurrent fever, anorexia, vomiting, colic, diarrhea, hematochezia and failure to thrive. All of the six cases tested for milk protein precipitin were positive. Pulmonary infiltrates were detected in all cases on radiologic imaging. One case had pulmonary hemosiderosis confirmed by iron laden macrophages in the bronchioalveolar lavage, gastric washing and open lung biopsy. The patient with pulmonary hemosiderosis recovered within a few months after elimination of cow's milk protein from the diet. The diagnosis of Heiner syndrome in all of these cases was based upon the improvement in the clinical and radiological findings after elimination of cow's milk protein from the diet. ${ }^{5}$ Elimination of offending food from the diet as early as possible is essential, as chronic pulmonary hemosiderosis results in changes in the lung such as fibrosis that can be fatal. Elimination of cow's milk from the diet has also been encouraged even without laboratory evidence of milk protein allergy due to poor prognosis. ${ }^{6}$ A short course of oral corticosteroids can be used for acute attacks. Patients usually outgrow this allergy and can tolerate cow's milk within a few years. ${ }^{5,7,8}$

\section{Conclusion}

This case report is presented to increase awareness of Heiner syndrome. Few cases were reported likely due to misdiagnosis and insufficient awareness of the disease. It is easily missed due to its resemblance to an infection or immune deficiency. Heiner syndrome is a rare syndrome but should be suspected in any unexplained pulmonary infiltrates or recurrent respiratory tract symptoms to avoid irreversible consequences that can be easily prevented by simple elimination of cow's milk protein from diet. ${ }^{9}$

\section{Acknowledgments}

The authors received no financial assistance for the research.

\section{Conflicts of interest}

There is no conflict of interest in composing this manuscript.

\section{References}

1. Sigua Jerome A, Zacharisen M. Heiner Syndrome Mimicking an Immune Deficiency. WMJ. 2013;112(5):215-219.

2. Mousan G, Kamat D. Cow's Milk Protein Allergy. Clinical Pediatrics. 2016;55(11):1054-1063.

3. Lee SK, Kniker WT, Cook CD, et al. Cow's Milk-Induced Pulmonary Disease in Children. Adv Pediatr. 1987;25:39-57.

4. Heiner DC, Sears JW. Chronic respiratory disease associates with multiple circulating precipitins to cow's milk. Am J Dis Child. 1960;100(4):500-502.

5. Moissidis I, Chaidaroon D, Vichyanond P, et al. Milk-induced pulmonary disease in infants (Heiner syndrome). Pediatric Allergy and Immunology. 2005;16(6):545-552.

6. Archer JM. Idiopathic Pulmonary Hemosiderosis Treated with a Milkfree Diet. Proc R Soc Med. 1971;64(12):1217-1218.

7. Eui Jung Lee, Douglas C Heiner. Allergy to Cow Milk. Pediatrics in Review. 1986;7(7).

8. Williams S, Craver RD. Cow's Milk-Induced Pulmonary Hemosiderosis. J La State Med Soc. 1989;141(8):19-22.

9. Boat TF, Polmar SH, Whitman V, et al. Hyperreactivity to cow milk in young children with pulmonary hemosiderosis and cor pulmonale secondary to nasopharyngeal obstruction. J Pediatr. 1975;87(1):23-29. 